\title{
REVISTAS EM EDUCAÇÃO FÍSICA E ESPORTES NO BRASIL: UMA BREVE REVISÃO
}

Ivone Job *

\section{RESUMO}

O texto apresenta uma síntese de revisão de literatura sobre a avaliação da pesquisa na área de Educação Física e Ciências do Esporte no Brasil. O mapeamento dos artigos refletem publicações dos últimos 30 anos com ênfase nas discussões da produção científica pela própria área, resgatados numa busca realizada no Google Acadêmico.

Palavras-chave: Revisão. Educação Física. Esportes. Indicadores de produção científica.

\section{INTRODUÇÃO}

Nos últimos anos tem crescido significativamente no Brasil a quantidade de publicações, no formato de artigos, livros, trabalhos apresentados em eventos e demais produtos em todas as áreas de conhecimento científico. Dados apresentados pela Thomson Reuters (PRODUÇÃO..., 2010) afirmam que a produção brasileira avançou de 3.665 para 30.021 artigos científicos publicados entre 1990 e 2008. O diretor da Springer Brasil, Harry Blom (2012), mostra que nossa produção cresce a uma taxa de $17 \%$ ao ano - enquanto a média mundial é de $3 \%$ - e já corresponde a $55 \%$ da produção científica da América Latina. Em outra reportagem Alisson (2012) comenta o novo Mapa da Ciência no Mundo em que países emergentes como China, Índia, Cingapura e Brasil expandem suas redes de colaboração em pesquisa e alteram a geografia tradicional comandada pelos Estados Unidos, França, Alemanha e Reino Unido. No Brasil, além da colaboração internacional entre autores, houve um aumento na formação de doutores. Somente em 2011 se formaram 12.000 novos doutores que escreveram 35 mil artigos científicos internacionais (ALISSON, 2012). Os Órgãos de financiamento de pesquisas observam esse crescimento, para verificar em que áreas deverão investir. Se por um lado podemos ficar orgulhosos da produção nacional, por outro, observamos que o número de citações aos trabalhos brasileiros, medido pelo Fator de Impacto (Fi) ainda é baixo, menor que a média mundial e também que quantidade não significa necessariamente qualidade. Atualmente há apenas dois periódicos brasileiros com Fi maior que dois: Clinics (2.058) e Memórias do Instituto Oswaldo Cruz (2.147).

Estes cálculos são obtidos com auxílio da bibliometria, ferramenta utilizada para medir indicadores de produtividade, que ajudam na análise e na avaliação das atividades da comunicação científica. Não deve ser utilizada indiferentemente, visto que cada área do conhecimento tem suas características, seus hábitos, seu comportamento, seu modo de escrever, suas fontes escolhidas, seus meios de comunicar as reflexões e experiências advindas de suas pesquisas. Assim, indicadores como Fi e Índice H, não podem ser interpretados 
unicamente pelo seu valor absoluto, mas em relação ao contexto das revistas e dos pesquisadores de sua área e de sua região.

Alguns termos provenientes da economia são utilizados nessas avaliações, tais como produção científica em que adotamos a definição de Lara (2006, p. 408):

[...] é a medida do volume de livros, capítulos de livros, artigos de periódicos e outras modalidades de produção impressas, digitais ou eletrônicas, contendo os resultados da pesquisa científica de autores, instituições, regiões, países ou áreas temáticas.

Indicadores podem ser definidos como as informações, provenientes de vários sistemas, susceptíveis de serem analisadas e formuladas para resolver questões específicas em um contexto específico. Assim, há indicadores de saúde, de economia, de segurança e os que aqui nos interessam são os indicadores bibliométricos que dão indícios a respeito da produção técnico-científica, segundo um determinado contexto: do país, de determinada base de dados, de pesquisadores, de uma área e devem ser interpretados junto com especialistas da área analisada.

Os indicadores utilizados nas avaliações realizadas pela Coordenação de Aperfeiçoamento de Pessoal de Nível Superior (CAPES) refletem diretamente sobre a pontuação auferida ao corpo docente e aos Programas de Pósgraduação (PPG) brasileiros. São fundamentados, principalmente, na produção de pesquisadores em revistas da área de educação física e esportes.

A Associação Brasileira de Normas Técnicas (ABNT) define revista como: "Publicação, em qualquer suporte, editada em partes sucessivas, com conteúdo corrente, designação numérica e/ou cronológica e destinada a ser continuada indefinidamente." (ASSOCIAÇÃO..., 2005, p.1).

$\mathrm{Na}$ área de Educação Física e Esporte segundo Ferreira Neto, (2006, p. 205) a revista científica: "[...] surge associada à pós-graduação stricto sensu e à criação de sociedades científicas a partir do final da década de 1970 as revistas Kinesis, Paulista de Educação Física, Motrivivência e Movimento." Característica comum no Brasil em que as revistas científicas são produzidas nas universidades e na maioria das vezes, sob a responsabilidade dos cursos de pósgraduação.

Atualmente, há no Brasil 21 Programas de Pós-graduação, modalidade acadêmico em Educação Física, conforme dados disponibilizados pela Coordenação de Aperfeiçoamento de Pessoal de Nível Superior (CAPES). Em 1990, há cerca de 20 anos, havia somente um doutor formado na área de educação física e hoje temos 162 doutores. Em comparação ao total da área das ciências da saúde, representamos em torno de 5,6\% de doutores formados nos últimos 20 anos. (ver Tabela 1) 
Tabela 1: Número de doutores da área de Educação Física e Ciências da Saúde, no período de 1990 a 2011

\begin{tabular}{lccc}
\hline Ano & $\begin{array}{c}\text { Número douto- } \\
\text { res/EF }\end{array}$ & $\begin{array}{c}\text { Ciências da } \\
\text { saúde }\end{array}$ & $\begin{array}{c}\text { Comparação (\% em } \\
\text { relação a Ci.Saúde) }\end{array}$ \\
\hline 1990 & 1 & 100 & 1 \\
2000 & 17 & 575 & 2,95 \\
2011 & 98 & 1.396 & 7,02 \\
Total & $\mathbf{1 6 2}$ & $\mathbf{2 . 8 9 0}$ & $\mathbf{5 , 6 0}$ \\
\hline \multicolumn{2}{c}{ Fonte: Dados obtidos em : PAINEL..., 2012 }
\end{tabular}

Certamente o número de pesquisadores em potencial e efetivamente afiliados a instituições de ensino da área de Educação Física é maior, considerando que muitos têm sua formação em nível de mestrado e de doutorado em programas de outras áreas de conhecimento, como por exemplo, na educação, na antropologia, na filosofia, na medicina, na fisioterapia etc.

\section{PROCEDIMENTO METODOLÓGICO}

A coleta de dados foi realizada no Google acadêmic (http://scholar.google.com.br/) o com a seguinte estratégia de busca: avaliaçao and produçao and educacao fisica and revistas and Brasil sem restrições de data de publicação. Esse resultado foi refinado pela leitura do título e do resumo, conforme a estrutura apresentada pelo Google Acadêmico, dos 100 primeiros registros, selecionando-se os que se referiam especificamente à temática educação física e avaliação. Assim, obtivemos os artigos aqui apresentados e analisados, em ordem cronológica de publicação.

\section{ARTIGOS MAPEADOS}

Dentre os artigos mapeados, a primeira publicação sobre o tema foi publicado no ano de 1992. Matsudo, (1992, p.68) levanta algumas características da produção: pertencente às Ciências do Esporte; enfatiza a necessidade de internacionalização da ciência brasileira; descreve as Ciências do Esporte como pertencente à "ciência Geral"; afirma que são baixos os padrões de cientificidade da produção; que há o problema da barreira da linguagem; critica a baixa produção brasileira em todas as áreas, pois de 20.000 trabalhos publicados há somente 2,51 de citações por trabalho, segundo dados do ISI de 1981-1990; afirma que "infelizmente" muitos cursos de EF no Brasil estão mais ligados a área de arte e não de ciências e que o nível do corpo docente de vários cursos, inclusive de Medicina do Esporte é precário. 
Vale a pena recordar que nessa época havia apenas 113 cursos de graduação em Educação Física, e duas revistas com periodicidade regular. Ao buscar soluções para os problemas apontados o autor sugere as seguintes alternativas: formação de recursos humanos na graduação e Pós-graduação; desenvolvimento de pesquisas com importância local, nacional e internacional; criação de um sistema integrado de informações; mais trabalhos com determinação de valores padrões para aptidão física, nutrição e atividade física. O autor alia uma boa produção à realização de pesquisas em aptidão física e índice de maturação biológica formando equipes de diversos esportes de alto desempenho.

Observamos que há vários pontos na sua análise, que refletem provavelmente um determinado período da história da produção da educação física em que a pesquisa era mais ligada às medidas em esporte. Por exemplo, o que se refere à afirmação de que é um problema a educação física estar ligada à área de artes? Parece que tal afirmação evidencia que nessas áreas não havia artigos no sentido de, não produzir cientificamente, não publicar. Noutro momento, o autor afirma de que teríamos que ser internacionais, no sentido de uma produção nacional de qualidade na área. Por outro lado, o autor cita a situação precária no Brasil e sugere alternativas importantes: formação de recursos humanos na graduação e na Pós-graduação; desenvolvimento de pesquisas com importância local, nacional e internacional e criação de um sistema integrado de informações.

Em outro artigo, França e Matsudo (1993) compara os artigos publicados na Revista Brasileira de Ciência e Movimento (RBCM) e na revista Research Quarterly Execise and Sport no período de 1987 a 1991. O autor apresenta os seguintes resultados: a RBCM teve mais citações a fontes "menos nobres" (p.1) e menor quantidade de referências atualizadas (com mais de 5 anos); salienta a adoção de resumos e títulos em inglês da revista brasileira como características com padrão internacional; quanto à autoria a maioria eram de autores pertencentes à editora da RBCM (48 autores), o Centro Estudos Laboratório de Aptidão Física São Caetano do Sul (CELAFISCS), (mas o autor não comentou a endogenia); denominou de "material pobre" as referências que não fossem artigo ou livro (p.13); e para suas análises nas revistas utilizou procedimentos como a análise paramétrica, variância, desvio-padrão.

No terceiro artigo mapeado (LUZ et al , 1996) os autores orientam os leitores/autores na elaboração das referências bibliográficas. Justificam a necessidade desse estudo a partir do seguinte argumento "[...] já que parodiando a conhecida máxima, do colega Laércio Elias Pereira, '[...] a partir de referências bibliográficas pobres, circulam pesquisadores medíocres'”. (p.6).

Os principais pontos que estruturam o artigo são no sentido de explicar e aplicar as normas da ABNT Norma Brasileira 6023 - Referências bibliográficas: procedimentos - NBR-6023 de 1989. No momento seguinte analisam e criticam as normas para autores das Revista Brasileira de Ciências do Esporte (RBCE) e Movimento apontando os acertos e as incongruências nas suas instruções. 
Concluem pela urgência de se atender a uniformidade dos dados das referências utilizando normas brasileiras a fim de uniformizar as informações de nossas revistas: "De fato, tais esforços não decorrem, como podem alguns julgar, de puro academicismo, mas sim de preocupação bem pragmática de recuperação de experiências e reflexões pregressas." (LUZ et al , 1996, p. 17).

Tal preocupação com a orientação de aspectos técnicos e formais dos artigos foi uma iniciativa válida e útil, pois vários editores começavam a dar maior atenção à normalização de suas revistas em função da padronização exigida pelas bases de dados indexadoras.

No quarto artigo analisado (TANI, 1998), o autor questiona o que denomina de questões seminais na área: as ciências do esporte têm estrutura acadêmico-científica? Qual a melhor denominação para identificar a área? Ele critica a "audácia" que recém-iniciados no mundo acadêmico científico tem ao publicar revisões de literatura sobre a Educação Física, fazendo avaliações críticas, resenhas e estado da arte, sem conhecimento suficiente da área e sugere que um periódico sério somente publique reviews realizado por renomados pesquisadores da área e geralmente a convite dos editores.

O ano de 1998 foi marcante para os programas de pós-graduação no Brasil em todas as áreas, porque foi adotado o Sistema Automatizado de Coleta CAPES. Com este instrumento a CAPES se provê de informações necessárias ao planejamento dos seus programas de fomento e delineamento de suas políticas institucionais. Nesse sistema foi incluída a avaliação Qualis para revistas brasileiras.

O sistema denominado Qualis (CAPES, 201, p. 1) é definido como:

[...] um instrumento de auxílio às Comissões de Avaliação para classificação dos veículos e eventos de divulgação da produção bibliográfica periódicos, jornais e revistas; congressos e eventos similares cadastrados pelos Programas de Pós-Graduação na Aplicação Coleta de Dados.

Esta classificação foi feita, inicialmente, em termos de circulação e qualidade: âmbitos internacional, nacional ou local e qualidade A, B, C. Desde então mudou a forma de hierarquizar os periódicos, mas ainda é um instrumento com extratos classificatórios de revistas fundamentado na publicação dos professores e do PPG. Após a implantação desse instrumento cresceu significativamente o número de artigos e revistas publicados na área de EF e esportes. $O$ que parece indicar a emergência de uma segunda fase da produção científica, alavancada a partir da publicação dos resultados da Avaliação Trienal de 1998-2000.

A partir deste período merecem destaque os trabalhos realizados pelo grupo Pró--teoria, coordenado pelos professores. Amarílio Ferreira Neto e Omar Schneider da UFES, em que destacamos:

Catálogo de Periódicos de Educação Física e Esporte (1930-2000), publicado em 2000 e apresenta, como o próprio título diz, uma lista de revistas brasileiras em educação física e esportes ordenadas cronologicamente por ano 
de criação. Cada uma delas com os sumários de todos os volumes e fascículos das revistas. É uma referência importante e trata com exaustividade o tema.

Pesquisa Histórica na Educação Física em 5 volumes: obra publicada em 2000 com ênfase na história da educação física no Brasil com destaque para o capítulo com bibliografia de mais de 400 referências sobre a educação física brasileira do séc. XX, especificamente no período de 1979-1999. Os autores apresentam uma justificativa para tal empreendimento que nos surpreende a de que as revistas eram pouco utilizadas para pesquisas naquele período, diferentemente do que ocorre atualmente.

A Revista Educação Physica: Estratégias editoriais e prescrições educacionais, (1932-1945): dissertação publicada em 2003, de autoria de Omar Schneider, pertencente ao grupo Pró-teoria. O foco do estudo é a revista Educação Physica publicada no Rio de Janeiro entre 1932 e 1945, por iniciativa de dois professores de Educação Física, Paulo Lotufo (editor no período de 1932 a 1945) e Oswaldo Murgel Rezende (editor no período de 1932 a 1944). Omar Schneider tem como objetivo analisar o aparelho crítico da revista e para atingi-lo especifica características como capa, periodicidade, layout, disposição do conteúdo, enfim características materiais, adotando a arqueologia do objeto amparado pela história cultural. Para Schneider (2003, p. 50):

[...] a palavra impressa não é apenas como registro do que aconteceu, mas como parte constituinte do acontecimento, como uma força ativa na história que registra ao mesmo tempo em que produz novas culturas, local em que se pode perceber a disputa entre o velho e o novo, em que bens culturais são produzidos, feitos veicular e apropriados.

Em 2002, o grupo Pró-teoria realiza avaliação de várias revistas de educação física no Brasil com instrumentos adaptados do formulário de avaliação de Krzyzanowski e Ferreira (1998) em seu estudo Avaliação de periódicos científicos e técnicos brasileiros, com algumas modificações no modelo original. Pela primeira vez vemos publicados trabalhos sobre as revistas brasileiras da área sendo analisadas por critérios bibliométricos. Justificam a necessidade de realizar o trabalho por consideram a pouca adequação dos periódicos científicos da Educação Física brasileira aos padrões formais adotados nacional e internacionalmente e assim pretendem contribuir para a melhoria da qualidade das publicações. Como exemplo desta produção temos os seguintes textos: Perfil de periódicos científicos da educação física: avaliação de aspectos formais (NASCIMENTO; GOMES, 2011) e Avaliação de periódicos científicos da educação física: avaliação de periódicos científicos da educação física (FERREIRA NETO, 2003). Outros trabalhos continuam sendo produzidos pelo grupo e seus participantes no sentido de avaliar a produção do conhecimento da área.

Em 2004 Kokubun responde ao artigo "Avaliação da Educação Física em debate: implicações para a subárea pedagógica e sociocultural", contestan- 
do algumas das análises feitas pelos autores e esclarece sobre a avaliação na pós-graduação em Educação Física pela CAPES. É a uma tréplica do seu artigo "Pós-Graduação em Educação Física: indicadores objetivos dos desafios e das perspectivas" de 2003 publicado em um número dedicado ao tema "pósgraduação" da Revista Brasileira de Ciências do Esporte (RBCE). Citando o trabalho de Guimarães e Gomes (2000) o autor oferece alguns dados importantes: a comparação de dados de produção intelectual da Educação Física com os de outras grandes áreas do conhecimento indicou:

1) dramática redução na proporção de artigos em periódicos com índice de impacto entre 1996 e 2000; 2) que 34\% dos docentes da pós-graduação em Educação Física nada publicaram entre 1998 e 2000; 3) proporção reduzida de trabalhos publicados internacionalmente, mesmo quando as bases de comparação eram a grande área de $\mathrm{Ci}$ ências Sociais e Aplicadas, Humanidades ou Letras/Lingüística/Artes; 4) o artigo apontou como falso o argumento de que, trabalhos que têm relações com aspectos socioculturais seriam predominantemente disseminados por meio de livros e capítulos, já que a proporção desses trabalhos nas grandes áreas de Humanidades, Ciências Sociais Aplicadas, Lingüística e Artes era menor do que aqueles publicados como artigos em periódicos. (KOKUBUN, 2004, p.197)

Estes argumentos eram para rebater uma discussão sempre presente na avaliação da produção da área, sobretudo para a área sociocultural. A baixa produtividade e o baixo grau de internacionalização foram apontados como problema crônico da área. Convém mencionar que o artigo original que desencadeou essa discussão foi elaborado com dados relativos ao final do triênio 1998-2000. O triênio seguinte, cuja avaliação foi concluída em 2004, foi marcado por intenso envolvimento dos programas no processo de avaliação da CAPES e que culminou com a criação de um Fórum de Coordenadores dos Programas de educação Física. Os dados da avaliação no período 2001-2003 revelaram o crescimento da produtividade docente, que duplicou entre 2000 e 2003 (de 1,9 para 3,8 autorias por ano), sobretudo em artigos completos, além de indicar um maior grau de internacionalização, leia-se, publicar em revista estrangeira (de $4 \%$ para $20 \%$ dos docentes publicaram pelo menos um artigo Qualis Internacional A ou B por ano).

Além disso, a Comissão de Avaliação da Área 21 da CAPES, em seu documento de área, apontou explicitamente a valorização da produção intelectual proveniente das subáreas socioculturais como um desafio a ser enfrentado. Estava em curso, um estudo para a elaboração de um Qualis Livro, com ampla participação dos programas de pós-graduação que foi aprovado posteriormente. O Conselho Técnico-Científico da Educação Superior (CTC-ES) du-

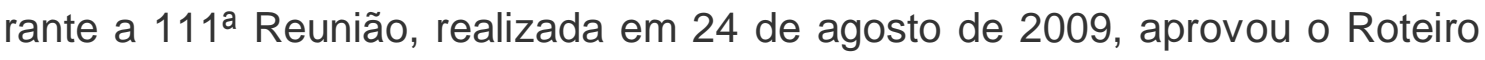
para Classificação de Livros. (CAPES, 2009). O roteiro traz conceitos e definições comuns e sugestão de modelo de ficha de classificação e servirá como orientação para as 23 áreas que vão classificar livros na avaliação trienal de 2010. 
A polêmica favoreceu a publicação de vários outros artigos. Em 2007, por exemplo, a RBCE publica artigos criticando a posição assumida pela comissão da área 21. Dentre eles destacamos os produzidos por Lovisolo, Rodrigues e Go Tani

Lovisolo (2007) parte do pressuposto que a área da Educação Física tem objetivo de intervenção orientada à realização de valores sociais e, no seu entender não é convergente com as orientações de avaliação impostas pela CAPES. Considera desmedida e irreal a regra de publicação estipulada no triênio (que ele denomina de "levantar o sarrafo") inviabilizando a elevação de qualificação dos PPG e de seus professores o que significa "dar um tiro no próprio pé". Ele considera particularmente mais difícil para as pesquisas de cunho social, humano da EF, cujas produções levam mais tempo para serem escritas, são mais locais e não causam tanto fator de impacto. Exemplifica com Paulo Freire que pouco escreveu em periódicos, mas é reconhecido e citado no mundo inteiro. Se fossemos avaliar realmente por qualidade de produção, diz ele, a inovação através de patentes deveria ser mais incentivada e o Brasil é muito pobre nesse quesito. Ao invés, a produção quantitativa em artigos funciona muito mais entre os pesquisadores, na base "é dando que se recebe", ou seja, o favorecimento de colaboração nas autorias sem o necessário envolvimento do pesquisador no trabalho.

Quase na mesma linha, Rodrigues (2007) afirma em seu texto que as transformações ocorridas nos últimos anos em termos de avaliação pelas agências de fomento causaram um aumento da produção científica brasileira, no entanto a pressão para aumentar quantitativamente essa produção pode levar a distorções. Metaforicamente, diz que um tamanduá-bandeira (pesquisador brasileiro) não sobreviveria, pois Ihe faltariam alimentos e recursos, já que neste sistema imposto pela CAPES, somente sobreviveriam os mais produtivos. Cita casos em que um pesquisador brasileiro considerado altamente produtivo pela CAPES, mas publica $50 \%$ de seus artigos em revista da qual é editor. Outro autor se utiliza de métodos nada éticos para aumentar sua produtividade: assina como autor intermediário em vários artigos com quem inclusive não é da área e cujo conhecimento não é sua especialidade, com a única finalidade de aumentar numericamente sua produção. Esses exemplos não contribuem efetivamente para o crescimento qualitativo da área, mas é como a CAPES quer, afirma Rodrigues (2007).

Contemplando argumentos em outra direção, Go Tani (2007) entende como necessário o constante aperfeiçoamento do sistema Qualis pois o identifica como uma forma de qualificar a publicação de revistas e assim, obter indexações representativas em bases de dados nacionais e estrangeiras. Recomenda a adoção de uma política de reduzir o número de revistas de educação física brasileira ao identificar que existe uma proliferação de periódicos, o que ele considera incompatível com o volume de artigos que a comunidade de pesquisadores da área é capaz de produzir. 
Um ano após essas publicações Silveira e Tani (2008) investigam através de editoriais da Revista Brasileira de Educação Física e Esporte da USP de 1986 a 2004 a trajetória da área de conhecimento de educação física no Brasil. Observaram que no período houve forte investimento institucional na produção científica acadêmica aumentando a visibilidade. Mas, concluem pela dificuldade de definir uma identidade acadêmica da área, que seria uma necessidade para a consolidação da área.

Numa linha de análise mais centrada na historiografia Souza et al. (2010) dividem o desenvolvimento da produção em educação física no Brasil em períodos. O primeiro equivale ao movimento originado em $1960 \mathrm{em}$ que houve uma necessidade de produção científica para justificar a EF como disciplina acadêmica; mesmo que essa produção fosse orientada aos interesses de outros campos do saber, denominados "ciências-mãe". O segundo seria a constituição da universidade dentro do modelo capitalista, que fomenta uma produção científica e cultural competitiva e orientada a certos interesses de uma elite econômica. Nesse contexto, a informação torna-se um produto que possui um valor e contribui para sustentar uma ideologia descomprometida com os interesses da área e do país e cria a rivalidade entre departamentos e os docentes. Na década de 1970 foi criada a pós-graduação no Brasil, mas os autores evidenciam as diferenças regionais em relação a essa política de desenvolvimento dos PPG e de financiamento público, com uma produção científica e tecnológica orientada pela lógica da produção capitalista de ciência. O autor chama a atenção para a grave discrepância regional no desenvolvimento da pesquisa e da pósgraduação em educação física no Brasil, com a inexistência de publicação de revistas fortes nas regiões nordeste e norte do país.

Entre outros trabalhos mais recentes desenvolvidos neste contexto encontram-se os publicados por Jacqueline Leta e Suely Rosa (2011) sobre análise da comunicação científica em EF, veiculada em quatro periódicos nacionais, no período de 2000 a 2005. Nesse texto as autoras analisaram as tendências e as características atuais da pesquisa brasileira em EF tendo como material empírico a produção acadêmica de 11 Programas de Pós-graduação disponível nos relatórios CAPES do triênio 2001/2003.

Observaram que: a pesquisa em EF no Brasil é realizada por pequenos grupos, preponderantemente de autores homens como primeiros autores, com concentração institucional, pouca colaboração, e pouca visibilidade fora do país. Além disso, há maior valorização de saberes advindos das ciências biológicas e, em especial, da fisiologia (são 376 publicações). Em minoria, estão as publicações de saberes procedentes de disciplinas mais humanísticas e/ou sociais, como a filosofia, a história, a sociologia, a psicologia, a educação, também são encontradas, porém, é ainda minoria, o que confere a heterogeneidade da área de conhecimento.

As análises de Suely e Leta têm como base os relatórios da CAPES de 2001-2003, portanto, passaram-se 10 anos, há de se olhar como uma análise de um determinado período da produção científica da área. As conclusões a 
que elas chegaram podem, hoje, não coincidir se realizarmos uma avaliação de um triênio mais recente, principalmente no que se refere à produção dos pesquisadores da área das ciências sociais e humanas.

Segundo dados da CAPES a produção da área em 2010 se concentrou em revistas de extrato $B 1$ e $B 2$, ou seja, nas nacionais mais bem avaliadas porque ao A1 e A2 correspondiam naquele ano somente revistas estrangeiras. Esse quadro se alterou em 2011 com o ingresso de revistas nacionais no extrato A2 como: Revista Brasileira de Medicina do Esporte, Movimento e Motriz.

Tabela 2: produção em artigos nas revistas segundo extrato Qualis, em 2010.

\begin{tabular}{|c|c|c|c|c|c|c|c|c|c|}
\hline \multicolumn{10}{|c|}{$\begin{array}{l}\text { Artigos completos publicados em periódicos téc- Total } \\
\text { nico-científicos }\end{array}$} \\
\hline 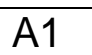 & $\mathrm{A} 2$ & B1 & B2 & & & & & & \\
\hline 216 & 305 & 86 & 8 & 2 & & & & & \\
\hline & 8,5 & 4,1 & 2,1 & 7, & 17,6 & & & & \\
\hline
\end{tabular}

Fonte: Dados obtidos em: http://www.capes.gov.br/

Nestes 10 anos, algumas revistas brasileiras foram aceitas nas bases WoS (ex ISI), Scopus e Scielo. Além disso, a Capes começou a pontuar produções em livro, o que vem a favorecer, de maneira muito justa, a pontuação de pesquisadores das áreas de ciências sociais e humanas.

\section{CONSIDERAÇÕES}

Este texto se propôs a fazer uma revisão do que foi publicado sobre a avaliação da produção científica em educação física no Brasil, nos últimos 30 anos. Este período não foi determinado no início do levantamento porque não se sabia exatamente quando começaram as discussões acerca da temática.

Longe de se esgotar a pesquisa, os documentos incluídos nesta breve revisão poderão ser ampliados, visto que o assunto ainda suscita e suscitará muitos debates entre os pesquisadores da área de educação física e esportes.

Podemos concluir que a discussão no período foi válida porque tivemos uma indiscutível melhora nas revistas da área, tanto no sentido formal quanto de conteúdo e de visibilidade, comprovada pela indexação em bases de dados reconhecidas internacionalmente.

Duas revistas estão indexadas em três bases de dados Web of Science (WoS), Scopus e Scielo: a Revista Brasileira de Medicina do Esporte e a Motriz. A revista Movimento, é indexada tanto na Scopus, na WoS e a única que 
está na base Redalyc, mas não está indexada na Scielo. As Revista Brasileira de Ciências do Esporte e a Revista Brasileira de Educação Física e Esportes estão indexadas em SciELO. Além disso, as revistas de Educação Física nacionais que foram incluídas em 2008 na WoS, a RBME e a Movimento já obtiveram o Fator de Impacto (FI) calculado em 2010, com 0.167 e 0.157 respectivamente, baixos ainda em comparação com revistas estrangeiras. Para Vilamon et al (2012) que analisaram as 5 revistas brasileiras e espanholas em educação física e esportes indexadas na base WoS, este fato tem um especial significado porque se trata das BdDs mais seletivas e prestigiosas que existem e em que se encontra a bibliografia científica de maior difusão, visibilidade e impacto internacional.

As contribuições dos textos apresentados ainda repercutem e nos deixam várias propostas, para termos uma área de pesquisa fortemente sedimentada em alicerces científicos, como a colaboração entre os pares, seja no âmbito institucional, nacional e internacional.

\section{REFERÊNCIAS}

ALISSON, Elton. Brasil integra o novo mapa da ciência. Agência FAPESP, São Paulo, 19 out. 2012. Disponível em: http://agencia.fapesp.br/16351. Acesso em: 5 nov. 2012.

ASSOCIAÇÃO BRASILEIRA DE NORMAS TÉCNICAS. NBR 10525: Informação e documentação. Número padrão internacional para publicação seriada - ISSN. Rio de Janeiro : ABNT, 2005. iv, 6 p.

BLOM, Harry. Scientific Publishing in South America Chances and Opportunities. In: HOW TO WRITE FOR AND GET PUBLISHED IN SCIENTIFIC JOURNALS, 1.2012 .2 Disponível em: http://www.fapesp.br/eventos/2012/03/howtowrite/harry.pdf Acesso em: 3 nov. 2012

CAPES. Qualis periódico. Brasília, DF: 2012. Disponível em: http://www.capes.gov.br/avaliacao/qualis. Acesso em: 3 dez. 2012.

CAPES. Roteiro para Classificação de Livros: avaliação dos Programas de Pós-graduação aprovada na 111ạ. Reunião do CTC de 24 de agosto de 2009. Disponível em: http://www.capes.gov.br/images/stories/download/avaliacao/Roteiro livros Trie nio2007 2009.pdf 
FERREIRA NETO, A. Publicações periódicas científicas em Educação Física e esporte de instituições universitárias. In: DACOSTA, Lamartine. Atlas do esporte no Brasil. Rio de Janeiro, CNOFEF, 2006. p. 205-206.

FERREIRA NETO, A. Bibliografia sobre a teoria da educação Física em periódicos brasileiros. In: FERREIRA NETO, A. et al. Pesquisa histórica na Educação Física Brasileira. Aracruz, Facha, 2000. v.5, p. 151-192.

FERREIRA NETO, A; SCHNEIDER,Omar. (coord.). Catálogo de Periódicos de Educação Física e Esporte (1930-2000). Vitoria: UFES, 2000.

FRANÇA, Nancy Maria de; MATSUDO, Victor K. Rodrigues. Análise quantitativa da Revista Brasileira de Ciência e Movimento e da "Research Quartely". Rev. bras. ciênc. mov., Brasília, v. 7, n.1,3 -4, p. 9-17, jan./out. 1993.

GUIMARÃES, J. A.; GOMES, J. Pós-graduação 2001: Desempenho, objetivos dos desafios e das perspectivas. Infocapes, Brasilia, DF., v. 8, n. 4, p. 6-29, 2000.

KOKUBUN, Eduardo. A avaliação da Educação Física em debate: esclarecimentos . Revista Brasileira da Pós-graduação , Brasília, v. 1, n. 2, p. 195200, nov. 2004

KRZYZANOWSKI, Rosaly Fávero; FERREIRA, Maria Cecília Gonzaga. Avaliação de Periódicos Científicos e Técnicos Brasileiros. Ciência da Informação, Brasília, DF, v. 27, n. 2, p. 165-175, maio/ago. 1998.

LARA, M. L. G. . Glossário: termos e conceitos da área de comunicação e produção científica. In: POBLACIÓN, Dinah Aguiar; WITTER, Geraldina Porto; SILVA, José Fernando Modesto da. (Org.). Comunicação \& produção científica: contexto, indicadores e avaliação. São Paulo: Angellara, 2006. p. 387-414.

LOVISOLO, Hugo. "Levantando o sarrafo ou dando tiro no pé." Revista Brasileira de Ciências do Esporte, Campinas, 2007, v.29, n. 1, p. 23-33. 
LUZ, I., GONÇALVES, A., BORGES, V.. Referências bibliográficas em ciências do esporte: conhecendo e aplicando recomendações técnicas. Movimento, Porto Alegre, v.3, n.4, p. 6-17, 1996.

MATSUDO, Victor K. Rodrigues. Condições presentes e problemas da pesquisa nos esportes na América Latina do ponto de vista da cooperação internacional. Revista Brasileira de Ciência e Movimento, Brasília, DF, v.6, n.1, p. 29-46, 1992.

NASCIMENTO, Ana Claudia Silverio; Andreia GOMES, Anchieta de Oliveira. Perfil de periódicos científicos da educação física: avaliação de aspectos formais. In: CONGRESSO BRASILEIRO DE CIÊNCIA DO ESPORTE, 17., 2011, Porto Alegre. Anais... Porto Alegre: CBCE, 2011.

PAINEL Lattes: Evolução da formação de mestres e doutores no Brasil. Brasília, DF: CNPq, 2012. Disponível em: http://estatico.cnpq.br/painelLattes/evolucaoformacao/. Acesso em: 4 nov. 2012.

PRODUÇÃO científica do Brasil ultrapassa a da Rússia, diz estudo. BBC Brasil, Brasília, 27 jan. 2010. Disponível em: http://www.bbc.co.uk/portuguese/ciencia/2010/01/100127 brasil russia ciencia rw.shtml. Acesso em: 30 out. 2012.

RODRIGUES, Luis Oswaldo Carneiro. Publicar mais, ou melhor? O tamanduá olímpico. Revista Brasileira de Ciências do Esporte, Campinas, v.29, n. 1, p. 35-48, 2007.

ROSA, Suely; LETA, Jacqueline. Tendências atuais da pesquisa brasileira em Educação Física: Parte 2: a heterogeneidade epistemológica nos programas de pós-graduação. Rev. bras. educ. fís. Esporte, São Paulo, v.25, n.1,p. 7-18, 2011.

SCHNEIDER, Omar. Educação Physica: a arqueologia de um impresso. Vitória: UFES, 2003.

SILVEIRA, Sérgio Roberto; TANI, Go. Educação Física como área de conhecimento na Escola de Educação Física e Esporte da USP: um estudo da sua 
trajetória e constituição a partir do seu periódico institucional. Rev. bras. educ. fís. Esp. São Paulo, v.22, n.1,p.35-44, jan.-mar. 2008.

SOUZA Eduardo Rumenig, LUZZI Alexandre, PEREIRA. Benedito O fordismo acadêmico na educação física. Rev. Bras. Ciênc. Esporte, Florianópolis, v. 32, n. 2-4, p. 43-57, dez. 2010.

TANI, Go. 20 anos de ciências do esporte: um transatlântico sem rumo? Revista Brasileira de ciências do esporte, São Paulo, Número especial, p. 19-30, . $\quad$ set. $1998 . \quad$ Disponível em: http://www.rbceonline.org.br/revista/index.php/RBCE/article/view/800. Acesso em: 30 nov. 2012.

TANI, Go. Educação Física: por uma política de publicação visando à qualidade dos periódicos. Revista Brasileira de Ciências do Esporte, Campinas, v.29, n. 1, p. 9-22, 2007.

VILLAMÓN, Miguel, JOB, Ivone,VALENCIANO VALCÁRCEL, Javier; DEVÍSDEVÍS, José. Estudio comparativo de cinco revistas de Ciencias del Deporte indizadas en WoS. Revista de psicología del deporte, Illes Baleares, v. 21, n. 2, p. 281-287, jul./dez. 2012.

\section{JOURNALS IN PHYSICAL EDUCATION AND SPORT IN BRAZIL: A BRIEF REVIEW}

\section{ABSTRACT}

The paper presents a summary of the literature review on the evaluation of research in the area of Physical Education and Sports Science in Brazil. The mapping of the articles reflect publications in the last 30 years focusing in discussions of scientific production by the own area, recovered in a search on Google Scholar.

Keywords: Review. Physical education. Sports. Scientific publication indicators

\section{REVISTAS EN LA EDUCACIÓN FISICA Y EL DEPORTE EN BRASIL: UNA BREVE REVI- SIÓN}

\section{RESÚMEN}

El artículo presenta una síntesis de revisión de la literatura sobre la evaluación de la investigación en el área de Educación Física y Ciencias del Deporte del Brasil. El mapeo de los artículos refleja las publicaciones de los últimos 30 años, con énfasis en las discusiones de la producción científica del área, rescatados en una búsqueda realizada en Google Scholar.

Palabras clave: Revisión. Educación Física. Deportes. Indicadores de producción científica 